\title{
Criteri di selezione per surrenectomia laparoscopica o retroperitoneoscopica
}

\author{
Pietro Giorgio Calò ${ }^{1}$ • Gianlorenzo Dionigi ${ }^{2}$ \\ Accettato: 14 settembre 2020 \\ (c) Springer Nature Switzerland AG
}

Commento a:

Patient selection and outcomes of laparoscopic transabdominal versus posterior retroperitoneoscopic adrenalectomy among surgeons in the collaborative endocrine surgery quality improvement program (CESQIP).

A.P. Marrero, H.S. Kazaure, S.M. Thomas, M.T. Stang, R.P. Scheri.

Surgery (2020) 167:250-256

La surrenectomia transaddominale laparoscopica (TAL) o retroperitoneoscopica posteriore (RP) sono diventate le procedure standard per i tumori surrenali non invasivi, primari e metastatici negli ultimi 25 anni [1]. Contrariamente all' approccio RP, l'approccio TAL consente un ulteriore spazio di lavoro con riferimenti anatomici convenzionali degli organi $[1,2]$.

Vari studi presso singoli Centri hanno dimostrato che i risultati delle due procedure non differiscono in modo significativo l'uno dall'altro ma che il RP può offrire vantaggi per i pazienti con tumori più piccoli, indice di massa corporea inferiore (BMI), patologie bilaterali e con patologie addominali, operazioni addominali precedenti.

Lo scopo dello studio di Marrero e collaboratori è stato quello di esaminare se possono essere identificate differenze qualitative tra le due procedure endoscopiche sulla base del programma multi-istituzionale statunitense American Collaborative Endocrine Surgery Quality Improvement Program (CESQIP) [2].

$凶$ G. Dionigi

gdionigi@unime.it

1 Divisione di Chirurgia, Università di Cagliari, Cagliari, Italia

2 Divisione di Chirurgia Endocrina e Mininvasiva, Università di Messina, Messina, Italia

In dettaglio, sono stati valutati i dati presentati da 34 centri al programma CESQIP tra il 2014 e il 2018. Pazienti di età inferiore a 18 anni e operazioni per recidive sulle ghiandole surrenali sono stati esclusi. Le lesioni della capsula tumorale vengono valutate nel CESQIP se sono state descritte rotture capsulari macroscopiche durante l'intervento endoscopico.

Su un totale di 1.145 pazienti inclusi nel CESQIP, 833 sono stati valutati, 539 (65\%) con TAL, 294 (35\%) con RP. Un carcinoma del rene sincrono è stato diagnosticato prima dell'intervento solo in 3 pazienti $(0,4 \%)$. In relazione alle malattie sottostanti, la RP è stata scelta più frequentemente di TAL per i seguenti tumori: sindrome CONN (30 vs 24\%), ormone inattivo (44 vs 40\%) e tumori del lato destro (47 vs $37 \%$ ). Un TAL è stato eseguito più spesso per tumori più grandi (mediana $3,2 \mathrm{~cm}$ contro $2,4 \mathrm{~cm}$ ), feocromocitomi (27 contro $22 \%$ ), tumori CUSHING (39 contro 20\%) e tumori del lato sinistro. Il $62 \%$ dei centri ha eseguito esclusivamente TAL, il $12 \%$ esclusivamente RP e il $27 \%$ entrambe le procedure. Il numero mediano di operazioni per chirurgo all'anno era 8,8 (RP) e 7,5 (TAL) ( $p<0,001)$. Non c'erano differenze tra TAL e RP in termini di tempi di intervento, sito del tumore e tassi di complicanze totali, compresi quelli con un $\mathrm{BMI}>40$. Il rischio di rottura della capsula tumorale è stato osservato in un totale del $9,4 \%$, di cui il $60 \%$ erano metastasi, era significativamente più alto per PR $(12,6 \%)$ che per TAL $(7,6 \% ; p=0,4)$; il principale fattore di rischio erano $\mathrm{i}$ pazienti maschi $(p=0,4)$. Il RP è stato associato a un tasso di conversione inferiore all'approccio aperto rispetto al TAL.

Il presente studio multicentrico è il più ampio finora sull'argomento TAL vs RP e, soprattutto, valuta l'aspetto della frequenza delle rotture della capsula tumorale. Tenendo conto dei limiti della qualità dei dati in tali studi e della diversa esperienza con il RP meno frequentemente praticato, il TAL 


\section{Bibliografia}

ha mostrato un chiaro vantaggio per quanto riguarda il rischio di rottura della capsula tumorale, che si verifica nel $60 \%$ delle metastasi; sono stati osservati tumori primari di dimensioni anche di $1,2 \mathrm{~cm}$. Il sospetto di neoplasie e metastasi sembrano quindi essere indicazioni inadeguate per RP. Sfortunatamente, non è stato possibile valutare il rischio di feocromocitomatosi con entrambi i metodi [1,2].

Nota della casa editrice Springer Nature rimane neutrale in riguardo alle rivendicazioni giurisdizionali nelle mappe pubblicate e nelle affiliazioni istituzionali.
1. Dralle H, Ehrlich AK (2020) Selection criteria for laparoscopic or retroperitoneoscopic adrenalectomy. Chirurg 91(7):595

2. Marrero AP, Kazaure HS, Thomas SM et al (2020) Patient selection and outcomes of laparoscopic transabdominal versus posterior retroperitoneoscopic adrenalectomy among surgeons in the collaborative endocrine surgery quality improvement program (CESQIP). Surgery 167:250-256 